\title{
Investigating the Specific and Alternative Nature of Mental Mathematics Strategies: The Case of Systems of Equations
}

\author{
Jérôme Proulx \\ Laboratoire Épistémologie et Activité Mathématique \\ Université du Québec à Montréal
}

The practice of mental mathematics enjoys a great reputation in school classrooms, curricular discussions, and other educational spheres. Its capacity for provoking the emergence of a variety of strategies, and thus to promote the development of flexible ways of solving, is often thought of as one of its main advantages and contributions. This article investigates another potential contribution of the practice of mental mathematics: namely, its capacity to engage solvers in specific and alternative ways of solving. This is explored through examples of strategies engaged in for solving one system of equations task. These strategies are analyzed in order to illustrate the ways in which they represent specific and alternative solving routes, ones that point to and insist on elements rarely attended to in usual paper-and-pencil work. Deserving of further studies, this analysis on the specific and alternative nature of mental mathematics strategies opens the door to an enriched conception of what the practice of mental mathematics can offer to the school mathematics landscape, going beyond an improvement of paper-and-pencil work and looking toward an extension and enrichment of mathematical ideas.

Keywords: Mental mathematics, strategies, specificity, alternative solving, systems of equations

As Threlfall (2002) explains, being confronted with the emergence of a variety of strategies to solve tasks is commonplace in mental mathematics contexts, where no paper-and-pencil is allowed and short time constraints are given for solving. Whether it be about solving arithmetical tasks like '117 49', algebraic tasks like 'solve $\frac{2}{5} x=\frac{1}{2}$ for $x$ ', statistical tasks like 'find the mean of the distribution " $0,0,4,2,4$ ", and so forth, solvers come up with numerous strategies and ways of solving. However true, provoking the appearance of a variety of strategies, which can lead to the development of flexible ways in solving, is hardly a dimension restricted to mental mathematics contexts; and having varied approaches and being flexible in 
their usage is conceived of as a fundamental competence to develop in mathematics in general (see, e.g., Heinze et al., 2009; Rittle-Johnson \& Star, 2007a, 2007b). Thus, developing varied and flexible ways of solving tasks might not capture the whole story in terms of the potential contributions of mental mathematics practices, and it has been argued that solving mental mathematics tasks engages more than this.

Studies on mental mathematics often report that the strategies used to solve problems differ from those usually referred to in a paper-and-pencil context. For example, Butlen and Pézard (1992) report that the practice of mental calculations can enable students to develop economical ways of solving arithmetic problems that traditional paper-and-pencil contexts rarely afford. This is because the latter are often focused on techniques that are too time-consuming in a mental mathematics context. These economical strategies can be seen to open varied and alternative mathematical routes for handling and addressing concepts under study, as Alain (1932) said many years ago:

Mental calculations represent a brilliant and new aspect of our teaching. The teacher and even the students constantly invent new ways of running without failing. This sort of exercise is sane for the spirit. (p. 81, my translation)

Others, like Murphy (2004) or Threlfall (2002, 2009), have discussed the aliveness and the on-the-spot nature of mental mathematics, where strategies are developed and tailored for the problem at hand and often differ from the writing processes that are usually the focus in paper-and-pencil contexts. Based on her review of historical curricular documents where the practice of mental calculations was salient, Poirier (1990) adopts similar views. She underscores the fact that mental calculations might have their own processes, differing from regular written calculations, raising therefore the issue of the specificity of strategies engaged with in a mental mathematics context. This idea of specificity of strategies is pushed further in Reys and Nohda (1994), where it is asserted that mental mathematics strategies are to be seen as more than the mental application of algorithms, and thus need not necessarily be linked to paper-and-pencil strategies, each having their own processes. Mental mathematics strategies are thus discussed in terms of "higher-order thinking process[es] where the generation of a strategy is as important as the execution of the strategy" (p. 12). However, apart from these assertions and explanations, few illustrations or examples are offered about the specificity and alternative nature of these mental mathematics strategies.

These various assertions raise interest in knowing more about the nature of mental mathematics strategies. In particular, this leads to aim at investigating the ways in which mental mathematics strategies could be specific, and differing from or contrasting with usual paper-and-pencil strategies. The objective of this article is to initiate such an investigation about mental mathematics strategies. 
Investigating the specificity of mental mathematics strategies is important to better understand how is mental mathematics fitting in the actual school mathematics landscape, dominated mostly by paper-and-pencil work, and what it can offer to it. This is oriented by the following questions: In what ways are mental mathematics strategies specific to mental mathematics? And, if so, in what ways do mental mathematics strategies open different or alternative ways of solving and engaging with the tasks given?

In order to explore these questions, data gathered on systems of equations are used as an illustrative case. Paraphrasing Papert (e.g., 1972, 1986), the plan of this article is to develop some examples of the kinds of mathematical strategies in which solvers engage to discuss the issues alluded to about the specificity and alternative nature of strategies in mental mathematics. This is so, on the one hand, because without the examples and illustrations, abstract statements about the specificity of mental mathematics strategies cannot be expected to have more than a suggestive meaning. And, on the other hand, the descriptions of the examples and illustrations are easier to follow if the reader already has an idea of their underlying intentions.

\section{Theoretical Framework}

\section{Conceptualizing the Emergent and Local Nature of Mental Mathematics Strategies}

A number of researchers argue that when solving mental mathematics tasks, solvers do not engage in a linear decision-making process to select a well-known strategy and then apply it; what is often termed the selectionthen-execution hypothesis or the toolbox metaphor. For example, Threlfall $(2002,2009)$ strongly criticized the notion that solvers make a "choice" from a previously developed toolbox of predetermined strategies. Building on Siegler's (1996) work, who also questions the notion of conscious or mindful strategy choice when solving problems, Threlfall rather insists on the organic emergence and contingency of strategies in function of the tasks and particularly the solvers themselves (e.g., find what they understand, prefer, know, have experienced previously with these tasks, have confidence with; see also Butlen \& Peizard, 2000; Rezat, 2011 on these matters). The same is true for Murphy (2004), who refers to Lave's (1988) situated cognition perspective to conceptualize mental strategies as flexible emergent responses adapted to particular situations. Strategies are thus not conceived as predetermined and "chosen", but as emergent, contingent on the solver and the problem at hand within a specific context of solving. As Threlfall (2002) explains:

As a result of this interaction between noticing and knowledge each solution 'method' is in a sense unique to that case, and is invented in the context of the particular calculation - although clearly influenced by experience. It is not learned as a general approach and then applied to particular cases. The 
solution path taken may be interpreted later as being the result of a decision or choice, and be called a 'strategy', but the labels are misleading. The 'strategy' (in the holistic sense of the entire solution path) is not decided, it emerges. (p. 42)

Mental mathematics strategies are thus conceptualized as adapted responses, tailored to the tasks and emerging from interacting with them (see Proulx, 2013a, 2019). This can lead to the development, as mentioned above, of local ways of solving tasks, emergent at the moment of solving. As Butlen and Pézard (1990) explain, mental mathematics tasks make specific strategies emerge, ones that do not always have "universal" or general possibilities (i.e., for solving large classes of problems): solvers develop particular strategies, for solving particular problems, efficient in particular ways, locally. This was also discussed early on by Plunkett (1979), who refers to mental strategies as "fleeting and often difficult to catch hold of [as] variable [and] active" (p. 3, emphasis in original), which sensitizes one to the emergent production of strategies for this tasks, at that moment, in that context. Referring to Plunkett's work, Murphy (2004) explains that these strategies are seen as:

Invented 'on the spot' by the user for that calculation and may not even be remembered for future use [...] mental calculation strategies [are] seen as 'active' as they are created by the user to suit the numbers involved. (pp. 3-4)

This also relates to Zack and Reid's (2003, 2004) analyses of ways of solving in terms of "good-enough" strategies, that is, not optimal or universal, but functional for the time being in solving the tasks: "good-enough for the time being' is not only a good move, it is one which we make all the time [...] assigning a general meaning and then going on." (Zack \& Reid, 2003, p. 43). This offers a view to understanding what happens in the midst of solving mental mathematics tasks, because it represents how the solver keeps on, or "makes do", as Zack and Reid would say. Thus, emergent strategies generated in this mental mathematics context can be seen as (continually) good-enough for solving the given tasks, the goal being not about finding the optimal way of doing, but mainly to $d o$ it, to be able to enter into the task and generate a way of making sense of and solving it.

Along that line, in Proulx (2019), mental mathematics strategies were linked with and conceptualized through Nunes et al.'s (1993) work on oral strategies. This led to conceive of mental mathematics strategies as meaningmaking endeavors, strongly connected with the task at hand, locally focused on the information or data available, and putting forward elements in these tasks that are often left aside in favor of general methods of solving: e.g., uncovering patterns and regularities to orient the solving of equations (doubling, tripling, cancelling; see Proulx, 2013b; Proulx et al., 2017), pointing to graphical cues to operate on functions ( $x$-intercept, intersections, 
parallelism of curves; see Proulx, 2015), assessing characteristics of a distribution before calculating its mean (extrema, clustering and number of data; see Proulx, 2017). These elements put forward diverse and also different ways of making sense of the task at hand and of the concepts themselves under study. In this sense, mental mathematics strategies can, by their local connection to the task they attempt to solve, raise elements of these tasks that other more general strategies (e.g., in paper-and-pencil context) might not pay attention to. The localness of these strategies, here far from being a limiting attribute, can orient toward elements of interest in the tasks that would otherwise be less salient.

This conceptualization appears fruitful for better understanding the processes at play when solving mental mathematics tasks, and to theoretically ground the argument that mental mathematics strategies have their own processes, their specificities, that distinguish them from usual paper-andpencil strategies: an argument that is explored and investigated empirically in this article with data excerpts and examples.

\section{Articulating Dimensions of Mental Mathematics Strategies}

Various dimensions of mental mathematics strategies that are found in the literature (e.g., Boule, 2008; Butlen \& Peizard, 1990, 1992, 2000; Kahane, 2003; MJER, 2008) can be articulated to contribute to develop a finer sense of the potential specificity of these strategies; as well as offer an analytical frame to look into the nature of strategies engaged with in mental mathematics. One set of dimensions concerns reasoned computations versus automatized computations. Reasoned computations imply the elaboration of personal strategies, often non-standard and adapted to the task. Contrarily, automatized computations imply access to an immediate result through the use of mathematical facts or memorized procedures. An example of this is demonstrated when finding area, that is, between using the formula $\left(\frac{D d}{2}\right)$ for the area of a rhombus, versus cutting the figure into triangles to find and compare the areas (see Figure 1).

\section{Figure 1}

Cutting the Rhombus to Find the Area

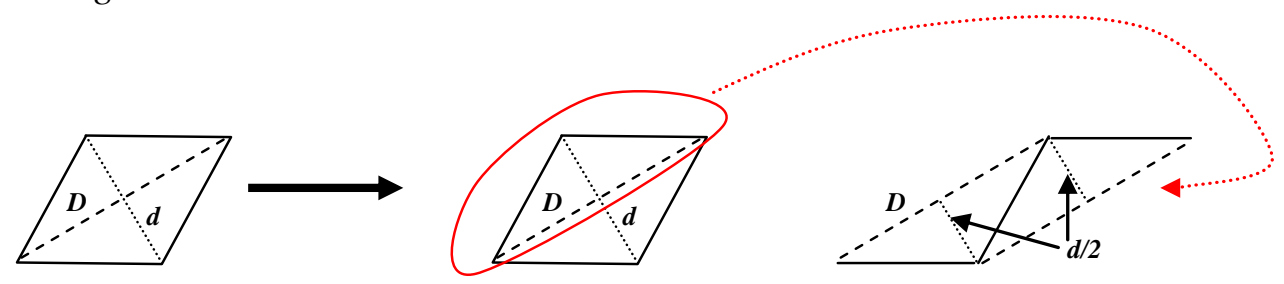

Another example of this is in statistics, when calculating the mean of the distribution " $0,0,4,2,4$ ". Solvers can be referring to the usual formula 
('sum of data $=10$ ' / 'number of data $=5$ '), versus modifying the distribution by taking 2 from each " 4 " and transferring it to the " 0 " to get all data equal to 2 , the mean, through an equilibrium view of the mean.

A second set of dimensions concerns approximate computations, based on estimation and approximation to gain a range for the answer, versus the application of an algorithm or a fact to obtain an exact answer. In trigonometry, an example is seen between using the fact that $\sin 30^{\circ}=1 / 2$ versus establishing a visual assessment that the length of the opposite side of a $30^{\circ}$ angle is approximately twice the length of the hypotenuse (see Figure 2).

\section{Figure 2}

Visual Assessment of Lengths in a Triangle with a $30^{\circ}$ Angle

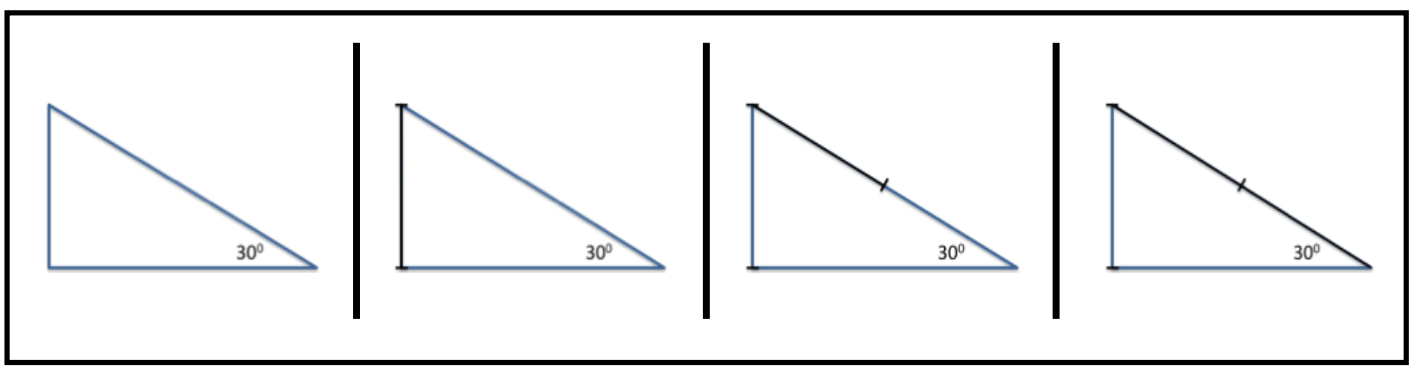

A third dimension concerns rapid computations, requiring a more global overview of the task to undergo quick executions to find the answer. Often criticized in mental mathematics because it is perceived as a speed exercise detrimental to sense-making, it can also be seen as helping to develop new solving methods. Rapid computations can force the solver, in trying to be economical, to abandon methods that may be slower (e.g., standard procedures) or less efficient for completing the task (e.g., one-on-one counting, technical symbol manipulations). In algebra, one example is the global reading of an equation (Bednarz \& Janvier, 1992) where for $x+\frac{x}{4}=\frac{x}{4}+6$ it can be directly asserted that $x=6$, thus avoiding numerous algebraic manipulations in order to isolate $x$. For an equation like $6 / x=3 / 5$ another global reading could lead to the assertion that because 6 is the double of 3 , then $x$ has to be the double of 5 , hence $x=10$, avoiding here again numerous error-prone algebraic manipulations (inverting, multiplying, dividing) in order to isolate $x$.

These dimensions illustrate how mental mathematics strategies can point to precise elements in tasks, which in turn raise alternative ways of solving and of making sense of these tasks (e.g., shortcuts, decomposition techniques, holistic views, approximations) that can extend ways of engaging with these tasks. These can reveal ways of making sense of and uncovering various elements of mathematical significance through the solving process (as in the examples above: cutting a shape in triangles, considering the overall 
structure of an equation, comparing and establishing ratios between lengths). These dimensions can contribute to drawing out meanings produced through these mental mathematics strategies, and to the investigation of the mathematical ideas engaged with.

\section{Methodological Considerations \\ Research Context and Participants}

In order to explore the research questions empirically, an analysis is conducted on strategies engaged in to solve one system of linear equations task in a mental mathematics environment. This example is taken from studies that are part of a larger research programme on mental mathematics, in which one of the objectives is to study the solving processes and the nature of the mathematical activity in which solvers engage. These processes are probed through a variety of group sessions, in elementary and secondary classrooms or in university pre- and in-service initiatives, where participants are asked to solve a number of tasks. The examined task in this article comes from a session on solving various systems of equations, conducted with adults taking part in one in-service initiative (from a daylong session, with 10 secondarylevel mathematics teachers from grades 9 to 12 ).

The participating teachers are regarded in this research programme as problem-solvers, as would any participant solving the given tasks in this research (be they elementary- or secondary-level students, (future) teachers, school principals, or even university students in mathematics). This being so, the decision to work with adult solvers is methodologically important. Indeed, these adults are not novice solvers of systems of equations and thus are not in a new solving context or familiarization with the topic. This enables them to be able to "enter" into the tasks to solve them, giving in return access to their strategies and mathematical activity. This might be otherwise with participants who are newcomers to the topic (e.g., Grade-9 students), as they might experience significant difficulties with the notion of systems of equations itself and could possibly not be able to enter into the tasks and solve them. In this sense, the intention is not to trace parallels between what these adult solvers do and what could happen with students in a classroom, nor is it to offer prescriptions for practice or to show how an approach through mental mathematics is "better" for learning about systems of equations than another focused on paper-and-pencil. The intention is to develop an understanding of the significance and functionality of the strategies brought forth when solving mental mathematics tasks (ways of engaging in tasks, elements points to, meaning given, etc.) and study the specificities of the strategies deployed, here for solving a system of equations task. This study is therefore purposefully exploratory, as a way into investigating the proposition that mental mathematics strategies can raise alternative and specific routes for solving, and to document this proposition with empirical examples. 


\section{Organisation of the Sessions and Data Collection}

The participants were sitting at large tables placed in a U-shape, facing the PI and the whiteboard. The general organization of the session is structured as follows: (1) a task is presented to the group orally or in writing on the board; (2) participants have approximately 15-20 seconds to solve the task; (3) at the PI's signal, participants are asked to write down their answers, e.g., on a small board given to them and to raise it for the PI to see; (4) strategies, adequate or not, are shared in a follow-up plenary, and notes are written on the board if needed to help understand them; (5) when solvers have no additional strategies to share, another task is offered and the cycle is repeated. For the examples of strategies examined here, participants had 15 seconds to solve the following system of linear equations: " $y=x$ and $y=x+2$ ". Then, they had to draw the intersection point on a sheet of paper displaying a Cartesian graph (with $y=x$ drawn as a reference line, see Figure 3 ), and raise it up for everyone to see. Strategies were then shared one after the other by the participants.

\section{Figure 3}

The Cartesian Graph Used to Report on the Solution

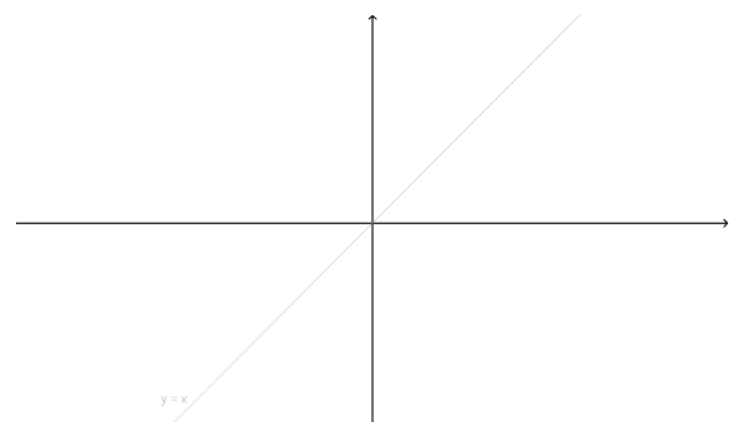

The data comes from the strategies orally explained by the participants (and subsequent drawings and notes made on the front board), recorded in note form by two research assistants, who afterwards collected and compared their notes to produce more substantial information about the strategies developed. The session was also video-recorded, which allowed the research team to return to the tapes to confirm, verify and enlarge on the notes and conduct analyses of the strategies.

\section{Data Analysis}

The analysis is focused, along the lines explained by Douady (1994), on the nature, meaning, and functionality of the strategies developed in this mental mathematics context in order to analyse the mathematical activity engaged with and study the specificities of the strategies. To achieve this, the previously outlined mental mathematics dimensions (reasoned, automatized, 
approximate, and rapid computations) are reinvested in the analysis. This enables not only to relate strategies with mental mathematics usual dimensions, but to use them to point at specific attributes of the mental mathematics strategies engaged in.

These dimensions are combined in the analysis with the above conceptualisations of mental mathematics strategies as meaning-making endeavors, strongly tailored to the task at hand. This combination leads to pay precise attention to how the strategies are connected to the task to solve, its "local" particularities, and thus what mathematical meaning emerged from the strategies in relation to the system of equations at stake.

This analysis is also conducted through contrasting with algebraic methods used in paper-and-pencil context for solving systems of equations (comparison, elimination, substitution methods, as detailed, e.g., in Proulx et al., 2009). As participants explained during the session, when solving these kinds of system of equations task in paper-and-pencil contexts, they resort to algebraic manipulations and known methods for finding the intersection point. However, in this mental mathematics context, they asserted being hardly able to do this because the burden of algebraically manipulating without paperand-pencil support became too heavy, leading them as they explained to having to develop alternative ways of solving. This enabled particular and local elements paid attention to in their mental mathematics strategies to become more salient and underscored. The analysis focuses on this aspect.

\section{Findings}

In the following, the strategies engaged with are first described. They are then grouped and discussed in relation with the dimensions of mental mathematics strategies and the local mathematical elements they point to. This leads to an analysis in relation to their specific mathematical nature and the alternative routes they offer for solving.

Strategies Developed for Solving the System " $y=x$ and $y=x+2$ " Strategy 1: The Role and Influence of Parameters

One participant focused on the fact that both lines would normally cross at $(0,0)$ if the second equation were $y=x$. But then, paying attention to the fact that the second equation was not " $y=x$ and thus had a $y$-intercept of 2, "I lifted it up". Thus, the answer was elevated by 2 on the $y$-axis, giving $(0,2)$ as an answer. [Even if it is the y-intercept for $y=x+2$, the answer $(0,2)$ was not talked in terms of the $y$-intercept, but mainly as an elevation of the "initial" intersection point $(0,0)$ toward the "new" one at $(0,2)$.].

\section{Strategy 2: Finding a "Line" of Possible Solutions}

One participant's algebraic manipulations focused on looking for a common $x$ that gave the same answer for both equations $(x=$ ? \& $\quad x+2=$ ?). 
He found that $x=1$ was this common $x$. He then drew a vertical line at $x=1$ (see Figure 4), explaining that he did not have enough time to find the exact value of $y$, but that the solution was on that line because $x=1$ gave the same answer for both equations. This gave him an infinite number of possible solutions, within the restricted domain of $x=1$. Questioned about his strategy, he explained that he had not focused on finding the value of $y$. Both finding $x$ and finding $y$ were seen at this time as separate ventures, one focusing on $x$ and one on $y$ (even if they are the same, since substituting $x=1$ also gives the value for $y$ ).

\section{Figure 4}

The Solution $x=1$ Given for Solving the System " $y=x$ and $y=x+2$ "

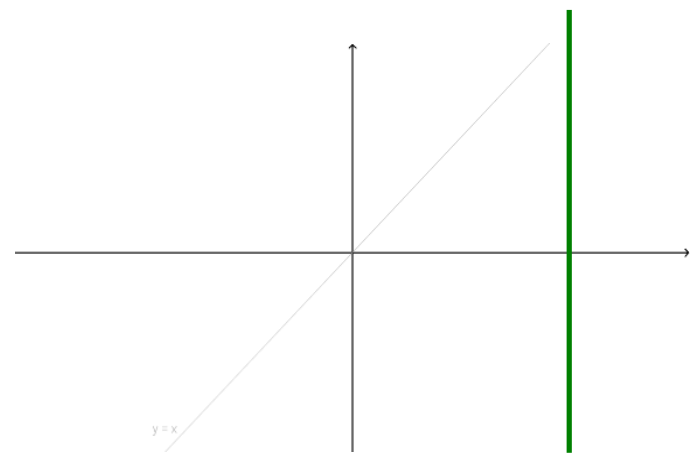

\section{Strategy 3: Visualizing in the Coordinate System}

One participant mentioned finding approximately where the point would be, by visualizing the lines as one that crosses the first quadrant in the middle $(y=x)$ and the other that goes through the $1^{\text {st }}$ quadrant as well $\mathrm{X}$ ( $y=x+2$, with a negative slope and starting from 2 on the $y$-axis), both intersecting on $y=x$ and in the $1^{\text {st }}$ quadrant. Visualizing the lines played an important role in positioning where the intersection point would be.

\section{Strategy 4: Visualizing the Lines with Objects}

Seeing pencils and pens on her table (see Figure 5), one participant imagined them as lines in the graph. She saw, similar to Strategy 3, that the intersection point was in the $1^{\text {st }}$ quadrant. It is through "seeing" the graph that the solution was developed, as this participant explained. 
Figure 5

Illustration of the Pen and Pencil on the Table Used to Imagine the Solution

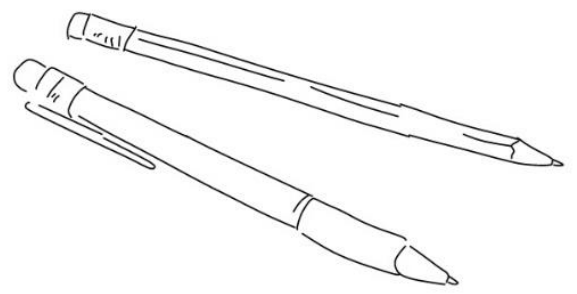

\section{Strategy 5: Finding the Right Quadrant}

For another participant, the first step was to realize that the solution would be in the $1^{\text {st }}$ quadrant, because of the equations of the lines in the system: one that splits the $1^{\text {st }}$ quadrant in half and the other that goes through it (although this participant was not necessarily precise in how the second line crossed the $1^{\text {st }}$ quadrant). Because of time constraints, the point was not placed precisely in the graph (close to the $x$-axis, a little to the right of the origin), and this participant knew this, but his main focus was on finding the right quadrant for the intersection.

\section{Strategy 6: The y-Intercept as a Focus}

Similar to Strategy 1, the $y$-intercept of 2 played an important role in determining the intersection point for one participant. This time it influenced the position of the line $y=x+2$, seen first as $y={ }^{-} x$, which had to be translated of 2 above. Thus, moving the line 2 above placed the intersection point higher than $(0,0)$ in the graph, "in that area" at the right of the $y$-axis, "but I did not have any precise value for it". [The point was placed somehow in mid-space between $(0,2)$ and $(1,1)$, without however aiming for any precise point.]

\section{Strategy 7: Visualizing the Lines with Gestures}

Similar this time to Strategy 4, one participant imagined placing and crossing hands to represent the slopes of the lines in the graph, which is a common teaching practice (see Figure 6). This gave her an idea of where the intersection point would be. This participant considered the point to be in the $1^{\text {st }}$ quadrant and then saw that the intersection point could be in the "middle" of the crossing hands (not necessarily realizing that the point was on $y=x$, with the same value for $x$ and $y$ as coordinates). Here too this participant explained that the solution was developed through seeing the lines. 
Figure 6

Crossing Hands to Represent Intersecting Lines

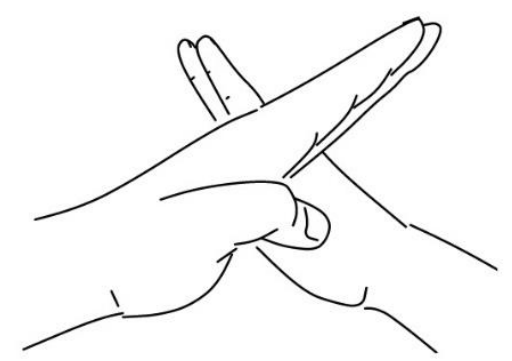

\section{Strategy 8: Trial and Error}

One participant explained having attempted a number of numerical solutions (however, not of the form $x=y$ ). But, because it led nowhere, he was unable to place any point of intersection (although knowing that there was one, because both lines did not share the same slope).

Analysis of Strategies for Solving the System " $y=x$ and $y=x+2$ "

Through their affinities and similarities, these strategies are grouped and analyzed in relation to the mental mathematics strategy dimensions. This leads to underline what they offer as alternative routes for solving this system of equations. Three routes are discussed: (a) situating the solution, (b) study of the equation, and (c) focus on shared numerical points.

\section{Approximate Computations: Situating the Solution}

For strategies 2, 3, 4, 5 and 7, the focus is on having a good idea of where the solution is in the graph and of what is happening in the system of equations. These strategies offer an analysis of the system, less for getting an exact answer than for obtaining a range, an "aroundness" of where the solution would be: whether by focusing on the value of $x$ as in Strategy 2, on the fact that the solution is on the line $y=x$ as in Strategy 3, on gaining a visual idea of where the lines intersect as in Strategies 4 and 7, or of knowing in which quadrant the solution is as in Strategy 5. The need to develop a sense of where the solution is situated appears quite remote from the algebraic methods (i.e., comparison, substitution and elimination) that aim to find the exact solution in $x$ and $y$ to the system through algebraic manipulations. These strategies underline aspects of interest to consider when solving a system of equations, mainly about visualizing the system and monitoring what is happening in it; a monitoring that the algebraic methods normally "do themselves" through the manipulations of the equations. The emphasis on $x=$ 1 in Strategy 2 and on $x=y$ in Strategy 3 are examples of how the focus on situating the solution offers significant information about the system: even if it 
seems obvious, the value of $x$ and the value of $y$ need to be the same for finding a solution that satisfies both equations, one of them requesting here that $x=y$ ! The same is true for the more visual Strategies 4 and 5 where lines were positioned, because one can directly see that there is an intersection point (something important to know about graphically for solving the system) and that the links between algebraic and graphical representations are salient. Hence one gains an idea of the system, of how it functions, and of what are its possibilities. It is in this sense that these strategies appear as specific for mental mathematics: not that they are better or worse, but mainly alternative, focusing on different elements of the task and offering another way into solving the system that a paper-and-pencil context does not necessarily lends itself to.

\section{Reasoned Computations: Study of the Equation}

Even if they give an answer that is mathematically inadequate, Strategies 1 and 6 demonstrate significant reasoning in finding a way to solve this system of equations relative to the value of the $y$-intercept and its influence on the solution to the system. These strategies are quite far removed from the usual algebraic methods. When solving algebraically, in a paper-andpencil context, the presence of the 2 in the second equation $y=x$, its $y$ intercept, is not given much consideration or seen as important as it is simply a " 2 ", the number "2", that is manipulated to find the value of $x$ or $y$ for solving the system (e.g., with the comparison method: $x=-x+2 \rightarrow 2 x=$ $2 \rightarrow x=1$ ). This is so since if the equation had been instead, e.g., $y=-x+$ 15 , this would have made no difference at the algebraic level in a paper-andpencil context (e.g., $x=-x+15 \rightarrow 2 x=15 \rightarrow x=7.5$ ). But, in a mental mathematics context, the impact of this 15 might have be tempered with quite differently by solvers ${ }^{1}$. Hence, in these algebraic manipulations, this " 2 " is not related to a " 2 " as the $y$-intercept, and the answer obtained is not conceptualized as being influenced by this "2"; any more than would the negative sign affecting the $x$ in the second equation (in algebraic manipulations, this negative sign is affecting an $x$, and does not saliently signify a "negative" slope for the solver as the previous manipulations with the comparison method shows). To the contrary, in Strategies 1 and 6, this "2" is the $y$-intercept and not simply a number to manipulate. Hence considerations of the "2" are not related to the mechanics of manipulating algebraically, but are for reasoning about the answer, that is, the point of intersection of both lines. Thus, in these two strategies, even if they do not offer the "right" answer, one finds that the equation is studied, its attributes are reasoned with and evaluated for finding something in it, for reading it so that it speaks directly to the solution; something the algebraic methods do not

\footnotetext{
${ }^{1}$ This example was suggested to me by Professor Andres Buchter in a conversation following the presentation of my work at the University of Duisburg-Essen, Germany, in February 2016.
} 
consider. In other words, these strategies offer a focus on differences in equations that make a difference for determining the solution.

In this sense, these two strategies call attention to precise and local elements of the equations, from the "-" sign affecting the $x$ to the " 2 " in the second equation, which makes the solving different than what could be done with other equations (e.g., $y=-x$ or $y=x+15$ ). The participants focused on the presence of the " 2 " in $y=-x+2$ to find the solution, the intersection point, which made it about that "2". However, the specificity of these strategies is not about the answer obtained or consideration of the "2", but rather about the effect that this "2" had on the answer: the fact that it influenced and played a role in the answer (e.g., 2 more, 2 higher). Strategies 1 and 6 are about reasoning on the effect of the elements of the equation on the solution to the system: something that is not of much significance or does not play a role in algebraic paper-and-pencil manipulations for solving.

\section{Bridging Automatized and Rapid Computation: Focus on Shared Numerical Points}

Strategies 2 and 8 focus on exact values for the solution by attempting to substitute possible answers to satisfy the system: a hybrid between automatized and rapid computations that aim to evaluate the system through working with its formula/equation and adapt to it. It can be said that these strategies are elementary, conceived only as attempts to try out possible answers in a trial-and-error and unsystematic venture. True as this may be, their interest however lies mainly in what they can make possible and orient toward: the solution needs to satisfy both equations simultaneously to be a solution to the system, a core notion for solving systems of equations. Indeed, if there were only one equation to satisfy right away, the numbers attempted would have given a solution, and this would be it (e.g., $x=2$ in $y=x+2$ would give $y=0$, and this would be satisfactory for an answer to this equation). But in the case of a system of equations, the solution needs to satisfy both equations simultaneously: both $x$ and $y$ need to be solution of both equations. The $x=1$ answer in Strategy 2 illustrates this, as $x=1$ is explained as a solution for both equations, meaning that it gives the same answer in each equation. (Again, the emphasis is on the fact that it gives the same "answer" and not the "same $y$ " for both, since the focus of the participant is on finding $x$ first.) The next step would have been to find the value of $y$ that is also the same for both equations, which would satisfy both simultaneously, but time did not allow for it. The same is true for Strategy 8, but here for coordinate points consisting of both $x$ and $y$ at the same time. The participant intended to rapidly test a value for $x$ and for $y$ at the same time in both equations and gaining a quick sense to see if both satisfied the equations (knowing that there was a solution, because they did not share the same slope, thus trying numbers to find it). These strategies, focused on shared numerical points, insist on the 
importance of finding a solution that satisfies both equations simultaneously through substituting simple values of $1,2,3$, etc., in its equations. With the use of algebraic methods, this intention to find values that satisfy both equations simultaneously remains implicit, hidden behind the algebraic manipulations of the equations. When that solution in $x$ and $y$ emerges, it does so at the end of the process, and not as a continual evaluation of the output that these numerical strategies engage in. These strategies made use of the simultaneous presence of both equations to try to satisfy them simultaneously. It is in this sense that these numerical trials appear specific in this mental mathematics context. ${ }^{2}$

\section{Additional Strategies Developed for Other Tasks}

In order to complement the previous analyses, two other types of strategies are discussed, ones that were engaged with for solving similar systems of equations. In detailing these strategies, the discussion also focuses on their specificities for solving.

\section{Global Reading: Analyzing the Lines Through Reading the Equation}

When solving the system " $y=3 x+1$ and $y=7 x$ ", one strategy was to globally analyse where the lines would be in the graph and then consider their intersection for the solution to the system. Thus one participant explained that $y=7 x$ passes by $(0,0)$ and is thus quite inclined, whereas $y=3 x+1$ "starts at 1", is less inclined, hence crosses $y=7 x$ at a $y$ greater than 1 and on the right of the $y$-axis (note that the analysis is made only in the $1^{\text {st }}$ quadrant, as this participant explained knowing where the lines would intersect from representing both lines graphically, whereas algebraic methods would not have directly offered the same information about the $1^{\text {st }}$ quadrant).

Another similar strategy was also related to the inclination of the lines, where the $y=7 x$ was seen as much more inclined, closer to the $y$-axis, than $y=3 x+1$, leading to a value in $x$ being between 0 and 1 for the intersection. Also, the value of $y$ was seen as greater than that in $x$ because $y=3 x+1$ has a $y$-intercept of 1 and a positive slope, leading the value in $y$ of the intersection to be greater than 1 . In both cases, the reading of the equation focuses on aspects that gives direct information about the lines in order to globally represent them for finding the solution. Understanding that the solution is in the $1^{\text {st }}$ quadrant because of the inclinations of the lines, that the value of $x$ is

\footnotetext{
${ }^{2}$ Links can be made to a guessing and testing arithmetic strategy in a paper-and-pencil context. This becomes of interest in terms of specificities when it is noted that these first arithmetic intuitions/strategies before engaging with and mastering the algebraic tools are mathematically quite powerful in relation to what solving a system of equations is and means. This understanding is often lost in the mechanical algebraic manipulations processes (see Sfard \& Linchevsky, 1994). Thus, the mental mathematics context can be said to bring back to the fore these central mathematical intuitions/strategies about systems of equations.
} 
between 0 and 1 , that the value of $y$ is higher than 1 , and so forth, do not appear to be a necessary part of the process one undergoes when using algebraic methods in a paper-and-pencil context, as these facts have little to no influence on the algebraic manipulations needed to get to the solution. In contrast, in this case, these elements are entailed in the strategies and take part in it.

In other cases of systems to solve, similar analyses were made of the line in the graph by reading the equation. For example, with the line " $y=x+10 "$, one participant said that it was parallel to " $y=x "$, but above it because of its $y$-intercept. Another example is with the system " $y=\sqrt{8} x+5$ and $\sqrt{18} x+3 y=9$ ", where one participant estimated the value of the slope of $\sqrt{8}$ as being close to $\sqrt{9}$ and thus close to $3 x$ with 5 as the $y$-intercept. For the second, the participant considered the $y$-intercept as being of 3 (from dividing 9 by 3 ) and thus the solution in $y$ as being between 3 and 5 in the $2^{\text {nd }}$ quadrant. In this global analysis, the participant explained that the intersection could not be lower than 3 in $y$, because it would then be in the $1^{\text {st }}$ quadrant, which is impossible because in that quadrant the other equation "starts" at 5 in $y$ (with its $y$-intercept being at 5). Thus, by being a negative slope that has a $y$ intercept of 3 , it had to intersect in the $2^{\text {nd }}$ quadrant and between 3 and 5 .

All these strategies represent specific ways of managing the solving of the system, performing a global reading of the equation, combined with a representation of the lines, for solving it. They offer a specific way into the systems, through globally understanding how they work and where and how the solution can be. These readings of the equation render some "information" in the equations as significant to solving the systems: something unusual in paper-and-pencil algebraic manipulations contexts.

\section{(Different) Approximation Computations: Approximate Algebraic Manipulations}

When asked to solve the system " $4 x+y=10$ and $x \quad 2 y=6$ ", one strategy was to opt for the elimination method by quadrupling the second equation, transforming it from $x 2 y=6$ to $4 x \quad 8 y=24$ and then subtracting it from the first (and obtaining $9 y=34$ ). Because of time constraints, the value obtained for $y$ was said to be around 5. Then, substituting the value of $y$ in the first equation led to a value for $x$ of around 1, for a coordinate point being about $(1,5)$. The degree of error in regard to the solution is significant, because there is a first approximation for $y$, and then one for $x$ based on that $y$. However, of interest in this strategy is the approximation of the values in using an algebraic method. This offers a range for both values, even if they are obtained through what is often seen as a precise and methodical method, that is, the algebraic elimination method. Whereas Strategies 2, 3, 4, 5, 7 for solving the system " $y=x \& y=-x+2$ " focused on gaining a range, an "aroundness" for situating the coordinate 
points in the graph, here it is in relation to algebraic manipulations: quite different from what is usually seen in an algebraic manipulation paper-andpencil context.

\section{Discussing Findings: What do we Gain from This?}

The analysis conducted on the mental mathematics strategies enables to see how mental mathematics strategies can be specific and offer alternative routes for tackling the tasks at hand. These mental mathematics strategies point to, and insist on, elements to which usual paper-and-pencil strategies often pay less attention to. It is in this sense that mental mathematics can be conceived as contributing in its own ways to the school mathematics landscape.

These strategies are no doubt a result of time constraints and because solvers cannot take notes, but this is the context of mental mathematics, and these are the kinds of specific entries that it generates and even promotes. As argued and documented in Proulx (2013a, 2013b, 2019), the mental mathematics context and its constraints create a need to grab something, to draw out aspects of significance for solving the task, which lead to numerous strategies that offer alternative routes to usual algebraic methods used in paper-and-pencil contexts. From this, a number of significant outcomes can be identified about the specificity of mental mathematics strategies, and the alternative routes they open for solving.

\section{Paying Attention to Specific Elements}

Through the examples, one can see how mental mathematics strategies can focus on elements not normally attended to in algebraic methods found in paper-and-pencil solving, hence bringing forth different ways of making sense and solving tasks. The example of focusing on the minus sign in $y={ }^{-} x+2$, or the assessment of the impact of the " 2 " on the value of the intersection point, that these lead to adequate solutions or not, are significant and illustrate how the strategies were kept close to the attributes of the task to solve and gave meaning to it (Proulx, 2019). These mental mathematics strategies underline how some precise elements about the tasks can be addressed when solving, developing strategies directly tailored to the task, somehow even transforming the task itself through all the alternative routes it offers in the process.

\section{Engaging in Good-Enough Strategies}

The analysis illustrates in concrete forms how in mental mathematics solvers engage in what Reid and Zack $(2003,2004)$ call good-enough strategies, where the solver's focus is on obtaining sufficient "information" to get a good idea and carry on the task for delivering a sufficiently close or coherent solution. Examples of this are illustrated by solvers aiming to find the right quadrant for the solution (through visualizing mentally or through artefacts like pens, pencils, or hands), as well as offering the line $(x=1)$ for the 
domain-solution of the system. In these strategies, one can see that precision is less of an issue than having a good sense of the solution, being able to situate it, offering solutions that are good-enough, close-enough, around-enough. Even if these strategies are not always optimal or do not render correct answers (precise or not), they offer alternative ways of engaging with the task; ways that differ from what is usually done with algebraic methods.

\section{Extending Possible Entries for Solving}

The analysis illustrates how these mental mathematics strategies bring forth alternative ways of making sense of tasks about systems of equations. These alternative meanings can be seen to be quite afar from ones gathered in algebraic methods; for example, about considerations of what happens in the equations themselves and how they behave. These various strategies are, to some extent, extending the ways the topic under study can be made sense of mathematically and the tasks solved. Through paying attention to precise elements, it shows possible ways of handling systems of equations; ways that would otherwise not necessarily have been as salient in algebraic methods, but are however constituting the core of these mental mathematics strategies (e.g., assessing the role of the $y$-intercept on the intersection point, viewing the inclinations of the lines, determining in which quadrant the intersection point is situated). The study of the equation and global reading strategies are examples of such. As some had stated (see Alain, 1932, or Reys \& Nohda, 1994 previously cited), mental mathematics prompts a way of inventing new and alternative ways of doing. Supporting these theoretical assertions, this study offers evidence of these "inventions", here in the context of systems of equations.

\section{Concluding Remarks}

The objective of this article is to explore the argument that mental mathematics can be conceived as specific and able to offer alternative ways of solving. The analysis of strategies reported here offers concrete examples, although initial, of that specificity of mental mathematics strategies. That mental mathematics strategies were specific and different than algebraic methods used in paper-and-pencil work is an argument that has been present in various documents, but for which there were not much, if any, evidence of. The examples of strategies examined in the context of systems of equations and the analysis conducted render support for these assertions by offering initial illustrations of these alternative routes and specificities found in mental mathematics strategies.

What results from this work about the specificity of mental mathematics strategies is the solidification of a conception of mental mathematics strategies as generating ways of solving that focus on aspects not usually engaged in or focused on in solving (e.g., here, the $y$-intercept, the 
boundaries where the value in $x$ and $y$ could be). These strategies are creative and exploratory, and they suggest extending what can be done with these topics: not in terms of the tasks offered, but in terms of the meaning to be given to the topic itself. These strategies bring us "elsewhere", where the focus is on other elements and toward other ways of solving than is usually aimed for in paper-and-pencil contexts.

The analyses conducted show how mental mathematics strategies can be said to venture into off-beaten tracks and back alleys, extending the scope of how topics under study can be addressed and tasks solved. They focus on, point to, and make salient local elements that algebraic methods used in paper-and-pencil contexts rarely necessitate or consider, through being general procedures needing to be efficient for solving entire groups of tasks. The local nature of strategies, raising alternative routes for solving, points to how mental mathematics has much to contribute to the enrichment of the school mathematics landscape. This appears to be a thread deserving of continuous attention and consideration in future studies.

\section{References}

Alain. (1932). Propos sur l'éducation. Les Presses Universitaires de France. Boule, F. (2008). Le calcul mental au quotidien. Cycles 2 et 3. SCÉREN, CRDP de Bourgogne.

Bednarz, N., \& Janvier, B. (1992). L'enseignement de l'algèbre au secondaire: une caractéristique du scénario actuel et des problèmes qu'il pose aux élèves. In A. Daïfe (Ed.), Actes du Colloque sur la formation des enseignants (pp. 21-40). ÉNS de Marrakech.

Butlen, D., \& Pézard, M. (1990). Calcul mental, calcul rapide. Grand N, 47, 35-59.

Butlen, D., \& Pézard, M. (1992). Calcul mental et résolution de problèmes multiplicatifs, une experimentation du $\mathrm{CP}$ en $\mathrm{CM} 2$. Recherches en didactique des mathématiques, 12, 319-68.

Butlen, D., \& Pézard, M. (2000). Calcul mental et résolution de problèmes numériques au début du collège. Repères IREM, 41, 5-24.

Douady, R. (1994). Ingénierie didactique et évolution du rapport au savoir. Repères IREM, 15, 37-61.

Heinze, A., Star, J. R., \& Verschaffel, L. (2009). Flexible and adaptive use of strategies and representations in mathematics education. ZDM Mathematics Education, 41, 535-540.

Kahane, J.P. (2003). Commission de réflexion sur l'enseignement des mathématiques - rapport d'étape sur le calcul. Centre national de documentation pédagogique.

Lave, J. (1988) Cognition in practice. Cambridge University Press.

Ministre Jeunesse Éducation Recherche. (2008). Les nouveaux programmes de l'école primaire. Mathématiques. Document d'accompagnement. Le calcul mental. Direction de l'enseignement scolaire. 
Murphy, C. (2004). How do children come to use a taught mental calculation strategy? Educational Studies in Mathematics, 56, 3-18.

Nunes, T., Schliemann, A. D., \& Carraher, D. W. (1993). Street mathematics and school mathematics. Cambridge University Press.

Papert, S. (1972). Teaching children to be mathematicians versus teaching about mathematics. International Journal of Mathematical Education in Science and Technology, 3(3), 249-262.

Papert, S. (1986). Beyond the cognitive: the other face of mathematics. In L. Burton \& C. Hoyles (Eds.), Proceedings of PME-10 (pp. 51-58). PME.

Plunkett, S. (1979). Decomposition and all that rot. Mathematics in Schools, $8(3), 2-5$.

Poirier, L. (1990). Évolution du role et de l'importance du calcul mental dans les programmes d'études québécois. Bulletin de l'Association mathématique du Québec, 2, 5-10.

Proulx, J. (2013a). Mental mathematics, emergence of strategies, and the enactivist theory of cognition. Educational Studies in Mathematics, 84(3), 309-328.

Proulx, J. (2013b). Le calcul mental au-delà des nombres: conceptualisations et illustrations avec la résolution d'équations algébriques. Annales de didactique et de sciences cognitives, 18, 61-90.

Proulx, J. (2015). Mental mathematics with mathematical objects other than numbers: The case of operation on functions. The Journal of Mathematical Behavior, 39, 156-176.

Proulx, J. (2017). Le calcul mental en mathématiques: Quels potentiels pour l'activité mathématique? Revue canadienne de l'enseignement des sciences, des mathématiques et des technologies / Canadian Journal of Science, Mathematics and Technology Education, 17(4), 288-307.

Proulx, J. (2019). Mental mathematics under the lens: strategies, oral mathematics, enactment of meanings. The Journal of Mathematical Behavior, 56, 1-16.

Proulx, J., Beisiegel, M., Miranda, H., \& Simmt, E. (2009). Rethinking the teaching of systems of equations. Mathematics Teacher, 102(7), 526533.

Proulx, J., Lavallée-Lamarche, M.-L., \& Tremblay, K.-P. (2017). Équations algébriques et activité mathématique en calcul mental: regard sur les défis d'enseignement. Annales de didactiqueet de sciences cognitives, 22, 43-65.

Reys, R.E., \& Nohda, N., with Shimizu, K., \& Smith, D. (Eds.) (1994). Computational alternatives for the $21^{\text {st }}$ century: Cross-cultural perspectives from Japan and the United States. NCTM.

Rezat, S. (2011). Mental calculation strategies for addition and subtraction in the set of rational numbers. Proceedings of CERME-7 (pp. 396-405). CERME.

Rittle-Johnson, B, \& Star, J.R. (2007a). Does comparing solution methods 
facilitate conceptual and procedural knowledge? An experimental study on learning to solve equations. Journal of Educational Psychology, 99(3), 561-574.

Sfard, A., \& Linchevsky, L. (1994). The gains and the pitfalls of reification The case of algebra. Educational Studies in Mathematics, 26, 191-228.

Siegler, R.S. (1996). Emerging minds: The process of change in children's thinking. Oxford University Press.

Star, J. R., \& Rittle-Johnson, B. (2007b). Flexibility in problem solving: the case of equation solving. Learning and Instruction, 18(6), 565-579.

Threlfall, J. (2002). Flexible mental calculations. Educational Studies in Mathematics, 50, 29-47.

Threlfall, J. (2009). Strategies and flexibility in mental calculation. ZDM The International Journal on Mathematics Education, 41, 541-555.

Zack, V., \& Reid, D.A. (2003). Good-enough understanding: Theorising about the learning of complex ideas (part 1), For the Learning of Mathematics, 23(3), 43-50.

Zack, V., \& Reid, D.A. (2004). Good-enough understanding: Theorising about the learning of complex ideas (part 2), For the Learning of Mathematics, 24(1), 24-28.

\section{Corresponding Author:}

Jérôme Proulx

Professor

Laboratoire Épistémologie et Activité

Mathématique

Université du Québec à Montréal

proulx.jerome@uqam.ca
Postal address

Jérôme Proulx

Departement de mathematiques

Université du Quebec à Montreal

C.P. 8888, Succ. Centre-Ville

Montreal, $Q c$

H3C 3P8 Canada 